\title{
NEURAL NETWORK BASED ENERGY EFFICIENCY IN WIRELESS SENSOR NETWORKS: A SURVEY
}

\author{
Neda Enami ${ }^{1}$, Reza Askari Moghadam ${ }^{1}$, Kourosh Dadashtabar ${ }^{2}$ \\ \& Mojtaba Hoseini ${ }^{3}$ \\ ${ }^{1}$ Faculty of Engineering, Payam Noor University, Tehran, Iran \\ nedaenamieyahoo.com \\ askariepnu.ac.ir \\ ${ }^{2}$ Department of Information Technology, Tarbiat Modares University \\ dadashtabaremodares.ac.ir \\ ${ }^{3}$ Department of Computer Engineering, Amirkabir University of Technology \\ mojtabahoseinidaut.ac.ir
}

\begin{abstract}
The main concern in Wireless Sensor Networks is how to handle with their limited energy resources. The performance of Wireless Sensor Networks strongly depends on their lifetime. As a result, Dynamic Power Management approaches with the purpose of reduction of energy consumption in sensor nodes, after deployment and designing of the network, have drawn attentions of many research studies. Recently, there have been a strong interest to use intelligent tools especially Neural Networks in energy efficient approaches of Wireless Sensor Networks, due to their simple parallel distributed computation, distributed storage, data robustness, auto-classification of sensor nodes and sensor reading. Dimensionality reduction and prediction of sensor data obtained simply from the outputs of the neural-networks algorithms can lead to lower communication costs and energy conservation. All these characteristics show great analogy and compatibility between wireless sensor networks and neural networks. This paper aims to present the most important possible application of neural networks in reduction of energy consumption according to research studies have been done in the literature so far through a general classification of them.
\end{abstract}

\section{KEYWORDS}

Wireless Sensor Networks, Energy Efficiency \& Neural Networks

\section{INTRODUCTION}

With the appearance of microelectronics, as sensor nodes became cheaper, smaller and lower weight, their batteries became smaller too. The main and most important reason for Wireless Sensor Networks development was for continuous monitoring of environments where are difficult or impossible for human being to access or stay for a long time; Monitoring of environments like head of an active volcano, difficult terrain border lands, bridges, battlefields, roads, sluices etc. So, normally, there is often low possibility to replace or recharge the dead nodes as well. The other important requirement is that in most applications of WSNs, we need a continuous monitoring, so the lifetime and network coverage of these networks are our great concerns since the performance of WSNs severely depends on their lifetime. Therefore, energy conservation is a serious and critical issue in designing of WSNs with longevity. Energy conservation should be gained by wisely management of energy resources. The first step to reduce the energy consumption of WSNs is to know the most energy consuming 
parts of these networks which are important in choosing the appropriate method. Energy consumption of communication subsystem is much more than that of computation subsystem. It is shown that transmitting of a bit of data needs to same amount of energy as running of a few thousands of instructions [1]. So, there should be a trade off between communication and processing tasks. Energy consumption of radio in all modes of reception, transmit and idle is the same extent, while energy consumption of radio part is reduced at least an order of magnitude in sleep mode. Thus it is reasonable to turn off the radio as long as we can. According to specific application, sensory subsystem may be a considerable source of energy consumption. In this case, it should be considered in energy efficient approaches although it had not been greatly noticed so far. Instead many research studies around the world have been done to reduce the energy consumption of radio communications. Several energy conservation schemes have been proposed in the literature, while there are several survey studies on them, mostly focused on an especial layer of protocol stack; such as several MAC protocols that have been proposed in the literature and comprehensive survey studies on them as in [2,3] or several routing protocols and survey studies on them[4,5]. But more comprehensive survey studies on energy conservation approaches of WSNs with a different viewpoint have been presented in [1] and [6]. Authors in [1] also presented a perfect taxonomy which divides all energy efficient approaches into three main groups: duty-cycling, data reduction, and mobility based approaches (figure1).

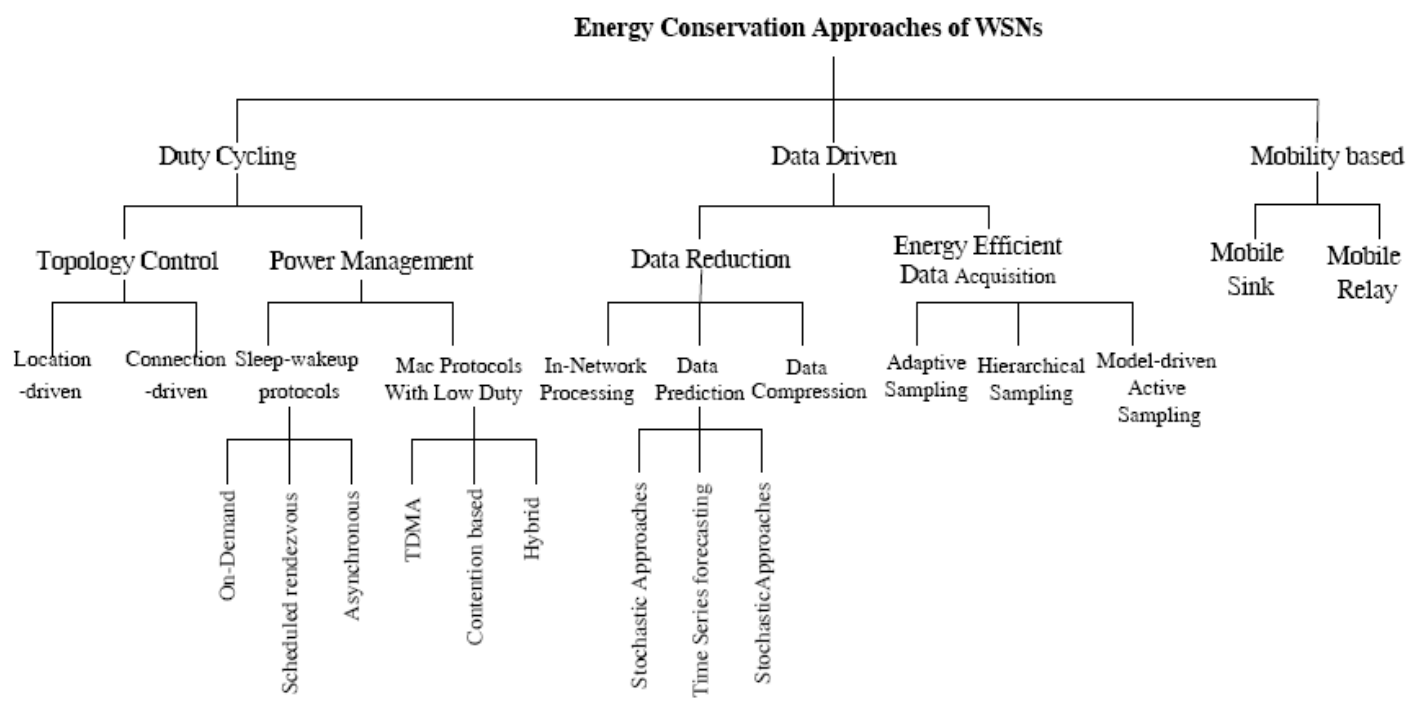

Figure 1. The taxonomy of approaches to energy conservation of WSNs [1]

Recently there is a strong interest to use intelligent tools such as Neural Network in energy efficient methods of Wireless Sensor Networks. Artificial Neural Networks are arithmetic algorithms which are able to learn complicated mapping between input and output according to supervised training or they can classify input data in an unsupervised manner. Some of the algorithms developed within the classical artificial neural networks, can be easily adopted to wireless sensor network platforms and in the same time they can meet the requirements for sensor networks like: simple parallel distributed computation, distributed storage, data robustness and auto-classification of 
sensor readings, fault tolerance and low computation. In a Wireless Sensor Network platform which has a fuzzy nature and different parameters are involved in it's behaviour, Neural Networks can help through dimensionality reduction, obtained simply from the outputs of the neural-networks clustering algorithms, leads to lower communication costs and energy savings[7]. Moreover due to centralized nature of WSNs in which all data from the sensor nodes often have to be sent to a (usually external) Base Station, Neural Networks capability in prediction of sensor readings at Base Station, can highly decrease unneeded communications and save considerable energy.

The other important motivation to use neural network based methods in WSNs is the analogy between WSNs and ANNs. As authors in [8] strongly believe that ANNs exhibit exactly the same architecture as WSNs since neurons correspond to sensor nodes and connections correspond to radio links. They also conclude that applying of the neural network paradigm in the context of sensor networks can lead to gain deeper understanding and more perceptions. With this view point, we can see the whole sensor network as a neural network and within each sensor node inside the WSN there could run also a neural network to decide on the output action. Therefore, efficient neural network implementations using simple computations can replace traditional signal processing algorithms to enable sensor nodes to process data by using fewer resources.

In our last work [9] we attempted to show how neural networks can help in different energy conservation approaches presented in [1]. In this paper we aim to present a new classification on Neural Network based energy efficient approaches based on their applications and appropriated neural topologies that had been applied for them in the literature. In table.1 you can see this classification. Then we will survey different methods according to that classification.

\section{NEURAL NETWORKS AND ENERGY CONSERVATION OF WSNS}

A Neural Network (NN) is a large system containing parallel or distributed processing components called neurons connected in a graph topology. These neurons are connected through weighted connections called synapses. Weight vectors (synapses) connect the network input layer to output layer. Indeed, the knowledge of NN is stored on weights of its connections and it doesn't need to any data storage. In other words, Artificial Neural Networks are arithmetic algorithms which are able to learn complicated mappings between input and output according to supervised training or they can classify input data in an unsupervised manner.

One of the difficulties with NNs is choosing of appropriate topology for the problem. This selection depends on properties of the problem, the most possible methods for solving the problem and also the properties of NN. Moreover there are different types of training rules which are inspired from biology science which determine the way NNs learn. In most of these networks, training is based on learning by example. Thus, a set of correct input-output data are often given to the network and using these examples, the network should change the weights values so that by inputting new data the network can return correct answers as output what we call "learning". One of the most important properties of NNs is ability to recognize the data affected by noise or intentional change and to remove those variations after learning. There are different types of NN's topologies, each have different capabilities according to the application needed. The 
network's capabilities depend on its structure, dynamics and training rules. The most important applications of $\mathrm{NN}$ include prediction, classification and identification.

The most important question is: how can Neural Networks help to energy conservation of Wireless Sensor Networks?

In fact, Neural Networks are not energy conservation methods and can not independently help to conserve energy but they can help energy conservation methods as intelligent tools to work in more efficient, desirable and easier way. So the energy conservation methods are the same previous methods which can use neural network as a tool to better approach to their goals. However there is enough motivation to implement full ANNs on each single sensor node due to analogy between WSNs and ANN as in [8].

Neural Network based energy efficient approaches can also be classified according to the role Neural Networks play on them or according to the appropriated neural topologies applied. In (table.1) you can see this classification where first column represents the role that neural network play in, second column represents the appropriate topology(ies) used for it with related references and third column represents the energy conservation approach category(ies) which it applied to.

Table 1. Classification of Neural Network Based energy conservation methods

\begin{tabular}{|c|c|c|c|}
\hline & The role N.N can play & N.N topology applied & $\begin{array}{c}\text { Energy conservation } \\
\text { category(ies) }\end{array}$ \\
\hline 1 & $\begin{array}{l}\text { Energy efficient } \\
\text { path discovery }\end{array}$ & $\operatorname{SOM}[12,13,14], \mathrm{BP}[15]$ & Duty cycling-routing \\
\hline 2 & $\begin{array}{l}\text { Energy efficient } \\
\text { Nodes clustering }\end{array}$ & $\begin{array}{c}\text { SOM } \\
{[16,17, \text { Our proposed EBCS }]}\end{array}$ & $\begin{array}{l}\text { Duty cycling- Cluster-based } \\
\text { routing }\end{array}$ \\
\hline 3 & Cluster head selection & SOM[20] & $\begin{array}{l}\text { Duty cycling- Cluster-based } \\
\text { routing }\end{array}$ \\
\hline 4 & Data aggregation/ fusion & $\mathrm{SOM}[8,26,33], \mathrm{BP}[34], \mathrm{RBF}[38]$ & $\begin{array}{l}\text { Data reduction-In network } \\
\text { processing }\end{array}$ \\
\hline 5 & Data association & Hopfield & $\begin{array}{l}\text { Data reduction-In network } \\
\text { processing }\end{array}$ \\
\hline 6 & Mobile data association & Competitive Hopfield & $\begin{array}{l}\text { Data reduction-In network } \\
\text { processing }\end{array}$ \\
\hline 7 & $\begin{array}{l}\text { Context /Data } \\
\text { classification }\end{array}$ & $\begin{array}{c}\text { SOM[8,22], MEMs[21] and } \\
\text { ART1[23] }\end{array}$ & $\begin{array}{l}\text { Data reduction-In network } \\
\text { processing }\end{array}$ \\
\hline 8 & Data prediction & $\begin{array}{l}\text { BP[25], Elman[24] } \\
\text { ARMA\& RBF[38] }\end{array}$ & $\begin{array}{c}\text { Data reduction-data } \\
\text { prediction and duty cycling }\end{array}$ \\
\hline
\end{tabular}

As you can see in table.1, some of neural networks capabilities can be contained in more than one category of energy efficient methods or can be a subset of other categories (e.g. data association is a subset of data fusion) but they have been mentioned separately for more emphasis. Also it is shown that Self Organizing Map neural networks obviously have more application in energy conservation methods compared to 
other neural topologies. Although this wide application of SOM can be explained with it's compatibility with multi dimensional data, self organizing and fuzzy nature of WSNs, more studies on other different neural network topologies are still required. In next sections, we will survey the most important neural based energy efficient methods which have been presented in the literature so far according to the role that neural network can play on them.

\section{ENERGY EFFICIENT ROUTING}

Today, radio communications are the most energy consuming task of WSNs [10, 11]. So many research studies focused on energy efficient routing protocols to address this problem. Routing is one of the most important issues of WSNs which can support performance of the network [4]. Due to energy constraints of each node in WSN, routing should be done in a way that balances the energy consumption among all nodes leading to extending the network lifetime and insuring network coverage.

\subsection{Energy efficient path discovery}

Due to the constraints on data processing and power consumption, the use of artificial intelligence has been historically discarded. However, in some special scenarios the features of neural networks are appropriate to develop complex tasks Such as path discovery [12].

The Self-Organizing Map (SOM) is an unsupervised neural network structure consists of neurons organized on a regular low dimensional grid [13]. Each neuron is presented by an $n$ - dimensional weight vector where $\mathrm{n}$ is equal to the dimensions of input vectors. Weight vectors (or synapses) connect the input layer to output layer which is called map or competitive layer. The neurons connect to each other with a neighbourhood relation as shown in (figure 2).

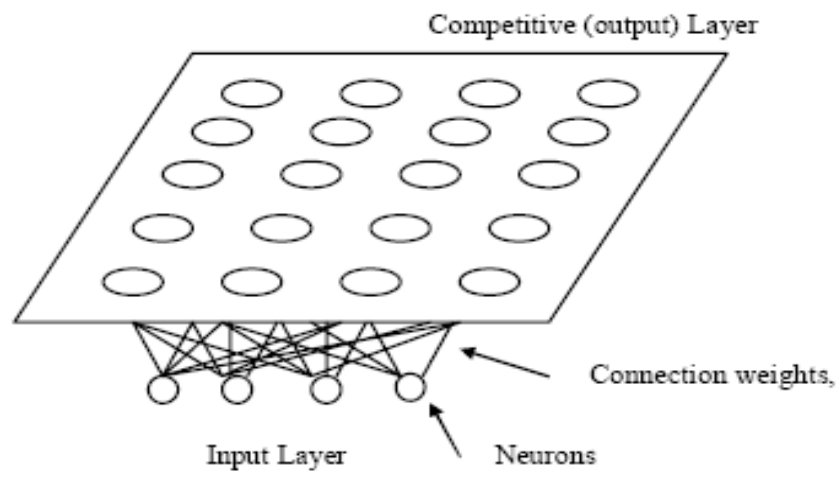

Figure 2. Self Organizing Map topology structure [16]

Every input vector activates a neuron in output layer (called winner neuron) based on its most similarity. The similarity is usually measured by Euclidian distance of two vectors. The important difference of a SOM training algorithm with other vector quantization 
algorithms is that not only the best matching units (the winner neuron) but also its topological neighbours would be updated. Close observations in input space would activate two close units of the SOM. The learning phase continues until the stabilization of weight vectors.

In [14] authors proposed an intelligent method based on Self Organizing Map neural networks that optimize the routing in the terms of energy conservation and computation power of each node. This algorithm has been designed for a wireless sensor node called MODABER created by artificial intelligence center of university of Isfahan. The assumption is that every node has an importance due to its role in routing so that the nodes which are used more than other nodes in routing have more importance due to their positions. They defined a Network Life Time (NLT) parameter which is sum of the nodes importance in routing at time $t$ and the amount of energy consumption of node for routing. They used a self-organizing (competitive) neural network to decide for every node containing the data packet and participate in routing or dropping the packet. The Self Organizing Map (SOM) learning algorithm is used for training of neural network. As soon as a packet arrives, its feature vector will be extracted and this vector is sent to self organizing NN of that node as input. The goal is to maximize NLT parameter. After winning of node in competition against other nodes, it is allowed to send the packet and participate in routing. Otherwise it should drop the packet. Since the learning algorithms of SOMs generally obey from linear computations, they believe that this method can be efficient to wireless nodes due to their limited computation and energy powers.

SIR [12] is another QoS-driven SOM based routing protocol in which a SOM neural network is introduced in every node to manage the routes that data have to follow. They proved that the inclusion of AI techniques (e.g. neural networks) in wireless sensor networks is useful tools to improve network performances.

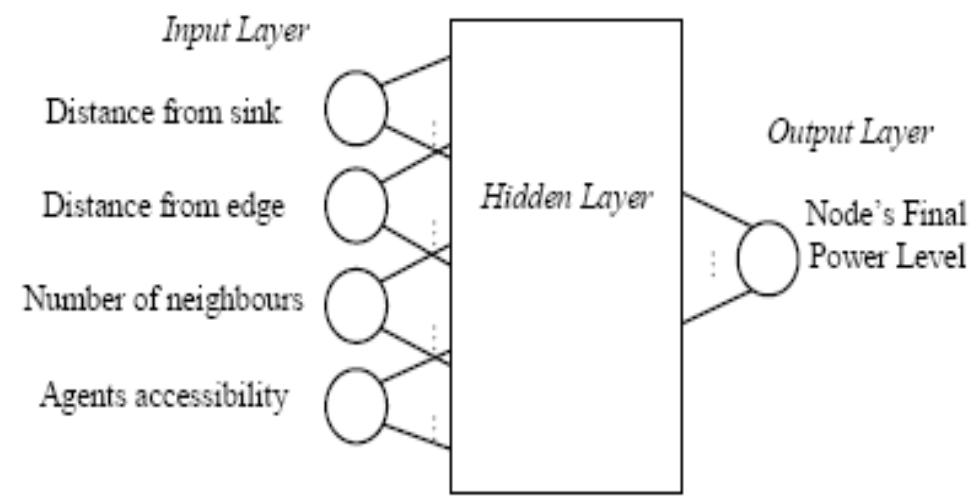

Figure 3. Back Propagation neural network applied in [15] to predict the final power level of the node

Usually a wireless sensor network life-time ends by having a single sensor node which uses all its power while other sensors have a significant amount of remaining power. The node which is in the routing path of many nodes to the base station is called a hotspot. In order to predict hotspots in a WSN, Authors in [15] defined a set of attributes for each sensor which were used as the inputs of our 3-layered back propagation neural network. These attributes belong to one wireless sensor node and by 
using them as the inputs of the neural network (figure 3). They can predict the powerlevel of the sensor at the end of WSN's lifetime. The predicted hotspots are then used in an Agent-based WSN route discovery and task management.

\subsection{Energy efficient nodes clustering}

Cluster based routing are the most frequently used energy efficient routing protocols in Wireless Sensor Networks which avoid single gateway architecture through dividing of network nodes into several clusters while cluster head of each cluster play the role of a local base station. Clustering algorithms often use the topological neighbourhood or adjacency as main parameter to form the clusters.

Low Energy Adaptive Connectionist Clustering (LEA2C) by Dehni et al. [17] is another LEACH-Centralized [18, 19] like SOM-Based clustering protocol. The cluster formation is done in a centralized way by Base Station. LEA2C uses a two phase clustering method, SOM followed by Kmeans as proposed in [13] (figure 4). The inputs to SOM are the coordinates of sensor nodes in network space. LEA2C applies the connectionist learning by the minimization of the distance between the input samples (sensor nodes coordinates) and the map prototypes (referents) weighted by an especial neighbourhood function. After set-up phase, the cluster heads of every cluster are selected according to one of the three criterions, max energy node, nearest node to BS and nearest node to gravity center of each cluster. Then the transmission phase starts and normal nodes send their packets to their CHs and on to the BS. In the case of using max energy factor for cluster head selection, the protocol would have a cluster head rotation process after every transmission phase. The transmission phase continues until the occurrence of first dead in the network. After that, the reclustering (set-up) phase will repeat. The simulation results show the profit of LEA2C over another LEACHbased protocol, called EECS [43] (In terms of 50 percent longer lifetime and insuring the network coverage during 90 percent of its total lifetime).

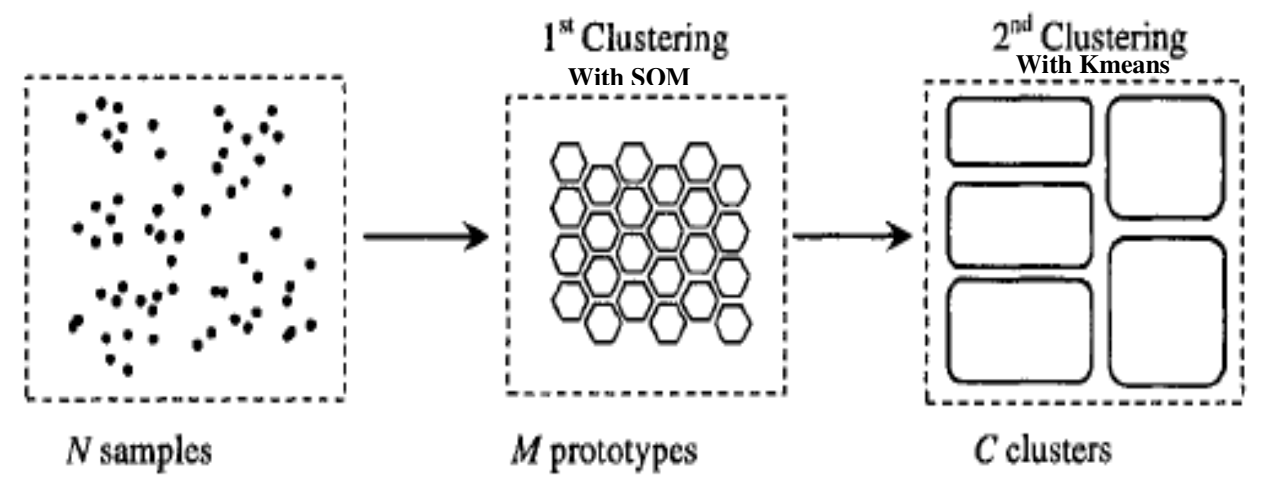

Figure 4. Two successive clusterings: SOM followed by K-means [17]

The authors in [16] used Kohonen SOM neural networks for clustering and their analysis to study unpredictable behaviours of network parameters and applications. Clustering of sensor nodes using Kohonen Self Organizing Map (KSOM) is computed for various numbers of nodes by taking different parameters of sensor node such as direction, position, number of hops, energy levels, sensitivity, latency, etc. 
SOM is an excellent tool for clustering of Wireless Sensor Networks because it is able to reduce dimensions of multi-dimensional input data and visualize the clusters into a map. Energy Based Clustering Self organizing map (EBC-S) [44] is a new topologicenergy based clustering method through using Self Organizing Map neural networks which can efficiently extend the network lifetime and network coverage. The reason for using SOM as preliminary phase is to make use of data pre-treatment (dimension reduction, regrouping, visualization...) gained by SOM [17]. Therefore the data set is first clustered using the SOM, and then, the SOM is clustered by kmeans. The variables to consider as SOM input dataset are $x$ and $y$ coordinates of every node in network space and the energy level of them. In fact, the variables of clustering are from two different types; distance and energy (figure 5).

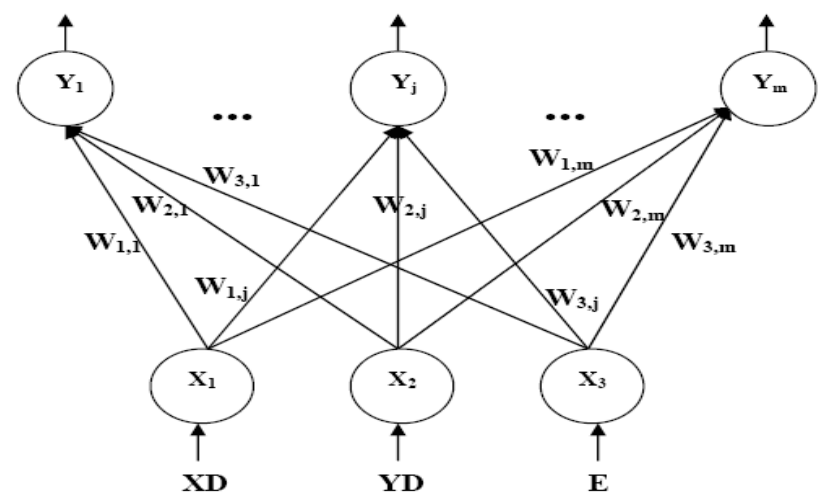

Figure 5. Self Organizing Map structure of EBCS protocol with three input parameters: node's coordinates and energy level

In order to determine weight matrix, Base Station has to select m nodes with highest energy in the network. We need three variables of these selected (high energy) nodes to apply them as weight vectors of our SOM: their x coordinates, their y coordinates and their energy level. The input samples should be first normalized with a min-max normalization method. The weight matrix of SOM consists of $X D=\left(x d_{1} \ldots x d_{n}\right)$ are $x$ coordinates, $Y D=\left(y d_{1} \ldots y d_{n}\right)$ are $y$ coordinates and $(1-E 1 / E m a x \ldots 1-E n / E m a x)$ are consumed energy of $m$ selected max energy sensor nodes. By making change in third variable (remain energy) of selected nodes, EBCS can move the nodes with less energy towards max energy nodes in order to form balanced clusters in the terms of energy level. The output of SOM is then given to Kmeans algorithm to reduce the number of clusters. The optimal number of clusters is also determined with Davies-Bouldin Index. In EBCS unlike LEACH and other clustering protocols, the boundaries of clusters are unlimited and each cluster does not necessarily contain adjacent nodes (as you can see in figure 6). EBCS algorithm can insure total survival (network coverage) during 95\% of network lifetime. Also the new algorithm can increase the lifetime of the network up to $50 \%$ over LEACH and $38 \%$ over LEA2C protocols. Moreover EBCS by insuring random and distributed dying of sensor nodes, show more network coverage compared with LEACH and LEA2C with the same number of alive nodes. 
International Journal of Computer Science \& Engineering Survey (IJCSES) Vol.1, No.1, August 2010
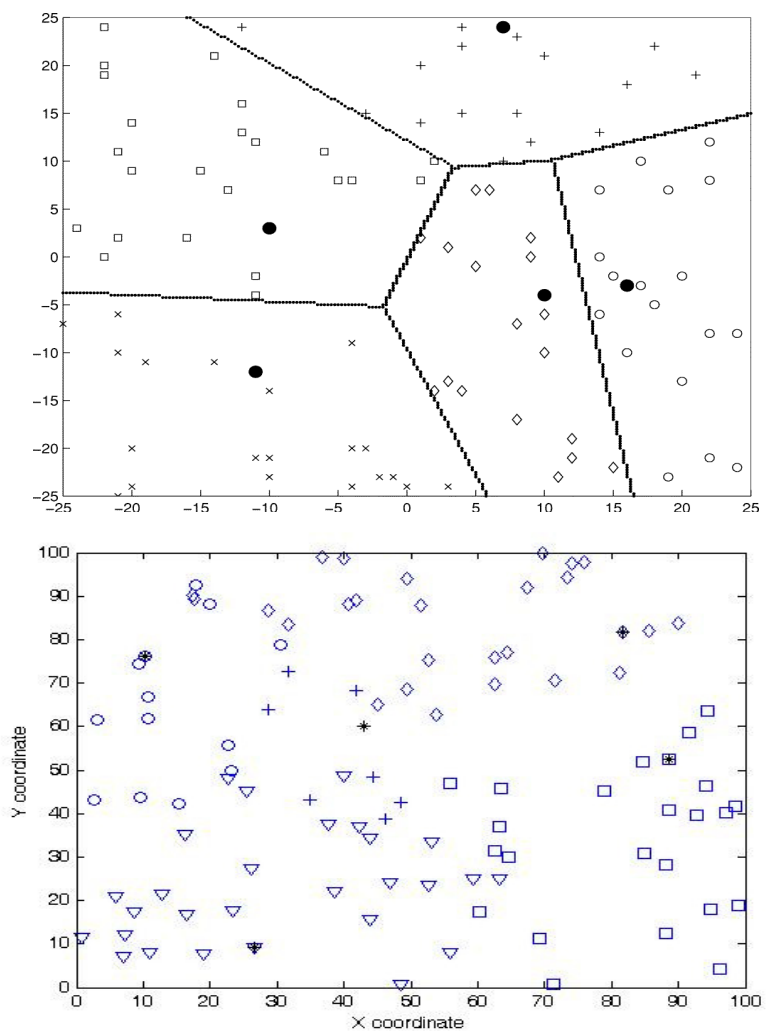

Figure 6. The cluster formation in (a) LEACH and (b) EBCS protocols. All nodes marked with a given symbol belong to the same cluster, and the cluster head nodes are marked with $\bullet$ in LEACH and with $*$ in EBCS.

\subsection{Cluster head selection}

Cordina and Debono [20] proposed a new LEACH like routing protocol in which the election of Cluster Heads is done with SOM neural networks where SOM inputs are intended parameters for cluster heads. SOM cluster the nodes according to their cluster head qualities. However a minimum separation filter should be applied on SOM output then to ensure a minimum separation distance between selected CHs (figure. 7). Results show a $57 \%$ profit of this protocol over LEACH (in the terms of first dead time). 


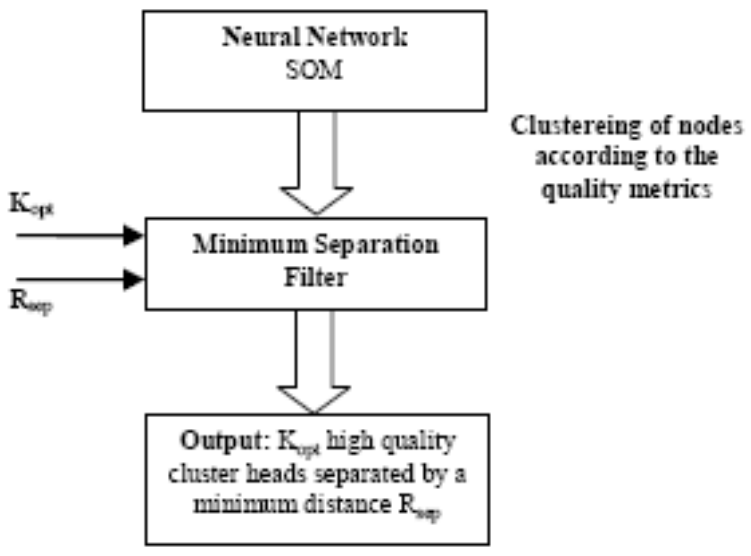

Figure 7. General operation of the SOM based cluster head selection mechanism [20]

\section{CONTEXT/DATA CLASSIFICATION}

Sensor nodes in an area usually form a sensing cluster and work together in a distributed and parallel way similarly to a layer of neurons. The data from all member sensors of a sensing cluster are from the same context but they are different because every sensor has a different point of view due to its different condition e.g. its position toward the event. Therefore these different data of cluster nodes have to be compressed and fused by in-network processing techniques. Such contextual information processing is carried out by ANNs as well

In [8] a SOM neural network has been used for reduction and classification of similar patterns. They used SOM in a hierarchical (cluster based) network architecture in which the nodes are organizes in several clusters with a cluster head or fusion centers. While reducing the amount of data to be transmitted, the SOM performs clustering of similar patterns. This characteristic enables the determination of relations between patterns which leads to their classification. This method can be applied in an event driven applications in where SOM can classify the event and increase the reliability of the decision.

Multiple elastic neural network modules (MEMs) [21] are an improvement to Self Organizing Map (SOM). MEMs generalize principles of self organizing model to enable management of wide range of complex optimization problems such as computer vision. Automatic context classification/recognition, usually by the analysis of measurement data from many sensor nodes, is a fundamental problem in humancomputer interaction. Generally, mapping the sensor data to a context is quite difficult because of the requirement of real-time classification and possibility of training patterns which contain sensor noise. In [22], context classifier based on Kohonen SelfOrganizing Map and online classification of sensor data has been studied.

In [23] researchers focused on using classification methods based on ART1 neural networks with the goal of reducing data traffic of node resulting in energy conservation. Sensor data which have too much redundancy, first, have to be classified by neural network in each node. Then, classified data were sent. In this way communication bandwidth increased efficiently. They used cooperative routing and also Directed 
Diffusion [45] for data routing. Both data routing results with and without classification have been compared in the terms of extending network lifetime.

\section{SENSOR DATA PREDICTION}

New sensing methods with energy efficiency through prediction of sensor measurements have shown great ability in reducing communications in sensor networks. In these methods, sink node extract model of time series to predict local readings instead of communicate with sensor nodes and receive actual measurements which consume too much energy. But most of methods were only limited to autoregressive linear models and considered for modelling of linear Phenomenon. Naturally, these linear models are inappropriate to estimate noisy multidimensional non-linear processes. In [24], authors proposed a frame integrated with non-linear time series models to approximate measurements and can reduce energy consumption by learning of a mapping that adapted with long lasting properties of needed process. As a result, it eliminates the demand for continual re-estimating of parameters of the model. Eventually, with reducing of communication among sink node and sensor nodes, energy consumption is minimized. Researchers used recursive Elman Neural networks to do so. Also they used methods based on second order Newton for training of the neural network.

Neural networks have been used in [25] for Dynamic Power Management of WSNs. The authors used NNs to schedule duty cycling of sensor nodes by event prediction. They proposed a neural method to decide which nodes and when have to be woken through prediction of the occurrence time of next event. They considered that the time of next event is a non-stationary series that can be predicted using Wavelet Neural Networks as accurate as possible. The neural network they used is actually a three layered Back Propagation which uses Morlet Wavelet transform at hidden layer (figure.8). The nodes which are at deeper sleep, consumes more energy to wake up. So state of the nodes can be determined with prediction of time series of next event and by defining a threshold relative to remained energy of nodes and comparing of those with each other. Simulation results showed that using the proposed method, energy consumption will be considerably reduced and total lifetime of wireless sensor network can increase considerably. The authors compared their proposed policy with last methods of Dynamic Power Management and gained better results. The results showed that with different rates of event lost, energy consumption will increase through their method. But still some open problems remain. First problem is that these methods do not analyze delay of the system due to waiting for sleeping nodes to wake up while delay is an important parameter in WSN. Secondly, there must be a solution for preventing nodes from loosing events while they are asleep and it should be efficiently managed. 


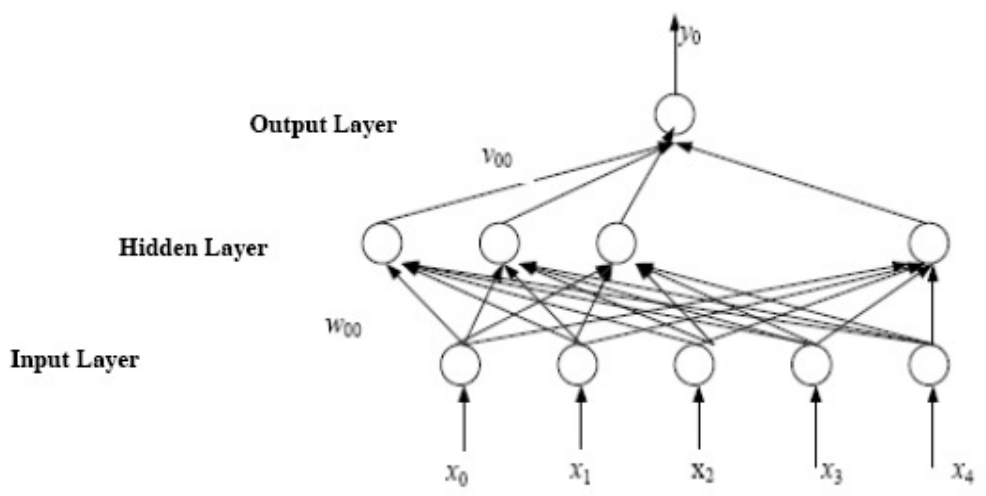

Figure 8. The Wavelet Back Propagation Neural network Topology Structure [25]

\section{SENSOR DATA FUSION}

Assuming that computation less than communication needs to energy, considerable energy conservation can be achieved through In-network processing especially data fusion. One of important issues of data fusion of WSNs is necessity of using an intelligent system which can fuse heterogeneous data obtained from different sources, accurately, automatically and efficiently. Data fusion can reduce the size of data. Moreover Even if the data had been affected by noise or intentional manipulating, data fusion method must be able to classify and identify the data. Sensor data fusion is a certain requirement of target detection and tracking applications in WSNs. One comprehensive survey study on sensor fusion approaches in target tracking is presented in [26]. One of the intelligent tools for data fusion is Neural Network. Sensor fusion with predefined numbers can be done with known methods such as Kalman filter or Bayesian theorem. But in cases that there is no specified statistical model for uncertainty (error estimation), instead, we should use other Intelligent methods such as rule based sensor fusion, Fuzzy Logic or Neural Networks [26]. Many authors [27], [28], [29], [30], [31] and [32] have successfully used neural networks in sensor fusion. Detection Statistics based on training algorithms of Artificial Neural Networks with actual or simulated values are trained to Neural Network. Neural Network can eliminate environmental or intentional jamming on sensory data according to training that they receive. Specific structures are considered for processing of detection statistics and sensors properties. Meanwhile heterogeneous multi sensor fusion is supported which requires the fusion of different data types.

In distributed systems, track to track association takes tracks which were created on multiple sensors and tries to associate or classify tracks that are related to the same target. This algorithm is NP-hard for more than two targets, and it needs to an approximate method to find the solution. A neural network method based on Hopfield structure proposed in [33] for this problem which always finds the optimal solution in 17.4 percent of the times and finds a way that approximate the proper solution in remained time.

Hopfield Neural Networks have a feedback from output to input. So they can provide a dynamic response. These networks may be unstable but stability can be supported with 
forcing the weight matrix to be symmetric with zeros along its main diagonal. A recurrent network contains an associative memory. Therefore, just like a human memory, if a part of this memory is supplied, the network can return the full memory. Associative nature of neural networks has been used to identify targets that we have very little information about them. In simple examples, these networks have no fault in identifying of objects so they can be applied to identification and classification of targets [26].

In [8] a Hopfield Neural Network has been used on every single sensor node to store the average correlations between components of all patterns. The lost or corrupted patterns can be recreated by HNN associative memory. The corrected or completed patterns can be used to build a data packets (as in figure. 9).

One of the earliest applications of neural networks multisensor data fusion for identification was in [34] which applied a Back Propagation neural network. In a Back Propagation Neural Network, the data is given to the network and the difference between the input and output is calculated. Weights are changed to improve the result. Once the errors have been minimized for all of the data in the training set, the system is ready to use for test data.

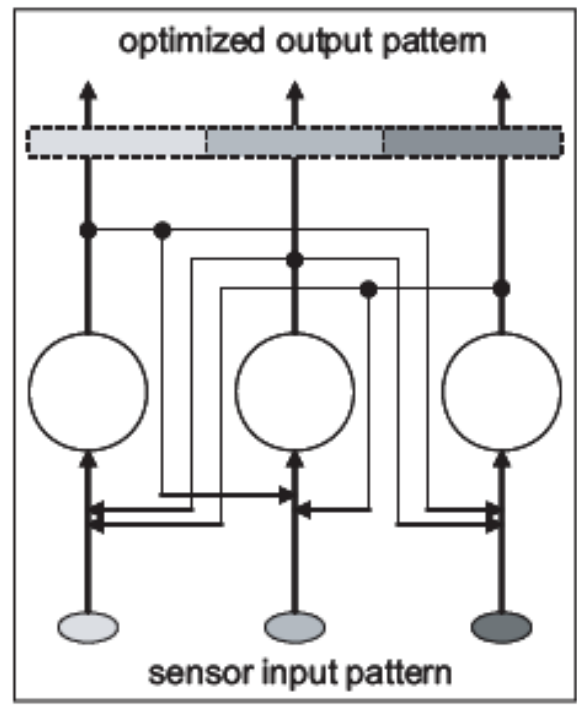

Figure 9. Hopfield neural network topology structure [8]

Multi-layer networks explicitly need long training time while Radial Basis Function neural networks (RBFs) (such as those which use networks with Localized Receptive Fields [35]) learn faster than Back Propagation networks because only one layer of weights needs to be modified. One of the most important problems of Multi layer neural networks is that determining of the number of appropriate hidden units is experimental. To solve this problem, Dynamic Node Creation (DNC) system [36] have been proposed which starts from a small network and increases the size one node at a time until the network size become large enough to do requested job.

In [37] energy efficient organization for WSNs with the purpose of target tracking is proposed. Target positioning through cooperative sensing can be achieved with multisensor data fusion. Last positioning results are used for adaptive prediction of 
target's trajectory. With composing of autoregressive moving average model (ARMA) and radial basis function neural networks (RBF), target position prediction can be efficiently done. Besides, by providing an efficient organization method, energy efficiency of WSN will increase. Authors in [38] proposed a new approach for optimal designation of duties by elastic neural network in tracking objects. First, a model of multi-coalition tracking multi-target is designed. Then disjoint fully connected sub graphs of neurons are constructed to solve the problem of optimized task allocation in tracking multi-target and increment of energy consumption when dynamic coalitions compete and conflict for the resources of sensor nodes. Compared with conventional methods, simulation results showed that the energy consumption of the tracking system was reduced significantly and the tracking accuracy improved greatly demonstrating the effectiveness of elastic neural network in handling optimized task allocation problem of multi-sensor multi-target tracking.

\section{MOBILE SENSOR DATA FUSION}

The mobile sensor data association in target tracking is one of most important techniques for WSN. The main issue in data association tracking algorithms is to partition the sensor data into sets of observations produced by the same target, and the other one is to avoid the couple effect exists between the mobile sensors for the same target. Data Association Algorithms (DAAs) consist of three parts: acquiring, processing and combining. Mobile sensor tracking with DAA is a prerequisite step for mobile sensor surveillance systems over WSN deployment [39].

There are several algorithms for DAA have been proposed for Multiple Target Tracking problem such as JPDA (Joint probabilistic data association) technique [40] which is appropriate for environments with high false targets. But these techniques may cause some unreliability or latency because all neighbourhood based methods usually consider the relations between sensor measurements and existing target tracks separately. So neural network approaches based on Hopfield Neural networks (HNN) have been proposed in [41] to solve this problem. HNNs which take weighted objective cost and constraints into an overall energy function are employed to combine with the neural network approaches to work out good tracking results. The difficulty for applying this method in DAA was that the determination of weight values was too difficult and it usually fell into irrational results. Recently researchers combined HNN with genetic algorithm, called HNN-GA [42]. They used this technique in a mobile based strategy in which using low network load and cooperation of mobile agents, it could optimize task allocations among nodes. In [39] the authors tried to take advantages of HNN so they improved the Competitive Hopfield Neural Networks (CHNN) algorithm which already had been applied in image processing applications. CHNN method can solve the above said problems by artfully managing of the weight updating function and the cost measurements. CHNN is an improved $\mathrm{HNN}$ in which a decision is made cooperatively. Each neuron receives information from other neurons and also gives information to others. With this collective information, each neuron goes to a stable state with the lowest value of predefined energy function. The global association of mobile sensor measurements and existing tracks will result in increasing the accuracy of mobile sensor tracking systems because in environments with dense targets the measurements 
produced by close targets can confuse the DAA algorithm and result in inaccurate relations. Moreover the competitive updating scheme of weights can solve the problem of determination of weight values said above, guarantee the convergence into a stable solution and avoid from falling into irrational solutions.

\section{Conclusions}

Energy conservation is the most important concern in Wireless Sensor Networks applications which should be considered in all aspects of these networks. Neural Networks as intelligent tools show great compatibility with WSN's characteristics and can be applied in different energy conservation schemes of them. This paper presented a classification for the most important applications of neural networks in energy efficiency of WSNs depend on different research studies have been done so far. The most important application of neural networks in WSNs can be summarized to sensor data prediction, sensor fusion, path discovery, sensor data classification and nodes clustering which all lead to less communication cost and energy conservation in WSNs. Another classification for neural network based methods can be according to neural network topologies that applied such as Self Organising Maps, Back propagation neural networks, recurrent neural networks, Radial Basis Functions etc. However, Self Organizing Map neural networks show more applications in WSN platforms.

As future work, more studies are required on different types of neural network topologies and training algorithms which would be more compatible with WSNs platforms in the terms of lower computation time.

\section{REFERENCES}

[1] Anastasi, G., Conti, M., Francesco, D.M, Passarella. A, (2009), "Energy Conservation in Wireless Sensor Networks: a Survey”, Ad Hoc Networks, Vol.7, No.3, pp.537--568

[2] Demirkol I, Ersoy C, Alagoz F, (2006) "MAC Protocols for Wireless Sensor Networks: a Survey”, IEEE Communications Magazine

[3] Langendoen K, (2008) "Medium Access Control in Wireless Sensor Networks", Book Chapter in Medium Access Control in Wireless Networks, Volume II: Practice and Standards, Nova Science Publishers,

[4] Al-karaki J.N, Kamal A.E, (2004) "Routing Techniques in Wireless Sensor Networks: A Survey”, IEEE Wireless Communication, pp.6-28

[5] Kemal Akkaya, Mohamed Younis, (2005) "A survey on routing protocols for wireless sensor networks”, Elsevier, Ad Hoc Networks 3, pp.325-349

[6] Shwe, H.Y., Xiao-hong, J., Horiguchi, S, (2009) “Energy Saving in Wireless Sensor Network”, Journal of Communication and Computer, Vol. 6, No.5, pp. 20--28

[7] Kulakov, A., Davcev, D., Trajkovski, G, (2005) “Application of wavelet neural-networks in wireless sensor networks", Sixth International Conference on Software Engineering, Artificial Intelligence, Networking and Parallel/Distributed Computing and First ACIS International Workshop on Self-Assembling Wireless Networks (SNPD/SAWN'05), pp.262--267

[8] Oldewurtel, Frank and Mahonen, Petri, (2006) "Neural Wireless Sensor Networks", International Conference on Systems and Networks Communications, ICSNC '06, pp.28 - 28 
International Journal of Computer Science \& Engineering Survey (IJCSES) Vol.1, No.1, August 2010

[9] Enami N, Askari Moghadam R, Haghighat, (2010) “A Survey on Application of Neural Networks in Energy Conservation of Wireless Sensor Networks", Recent Trends in Wireless and Mobile Networks, WiMo Proceedings, Ankara, Turkey, pp. 283-294.

[10] Pottie G, Kaiser W, (2000) "Wireless Integrated Network Sensors", Communication of ACM, Vol. 43, N. 5, pp. 51-58.

[11] Raghunathan V, Schurghers C, Park S, Srivastava M, (2002) "Energy-aware Wireless Microsensor Networks”, IEEE Signal Processing Magazine, pp. 40-50.

[12] Barbancho J, Leon C, Molina F.J, Barbancho A, (2007) “Using artificial intelligence in routing scheme for wireless networks", Computer Communications 30, Elsevier, pp. 2802-2811

[13] Vesanto J, Alhoniemi E. (2000) “Clustering of Self Organizing Map”. IEEE Transactions on Neural Networks, Vol. 11, No. 3, pp. 586-600.

[14] Shahbazi, H., Araghizadeh, M.A., Dalvi, M., (2008) "Minimum Power Intelligent Routing In Wireless Sensors Networks Using Self Organizing Neural Networks", IEEE International Symposium on Telecommunications, pp. 354--358

[15] Hosseingholizadeh Ahmad and Abhari Abdolreza (2009) "A neural network approach for Wireless sensor network power management"

[16] Aslam N, Philips W, Robertson W, Siva Kumar SH, (2010) "A multi-criterion optimization technique for energy efficient cluster formation in Wireless Sensor networks", Information Fusion, Elsevier

[17] Dehni L, Krief F, Bennani Y., (2005) "Power Control and Clustering in Wireless Sensor Networks", Challenges in Ad Hoc Networking, vol 2005, pp.31-40.

[18] Heinzelman W, Chandrakasan A, Balakrishnan H., (2002) “Application-specific protocol architecture for wireless microsensor networks", IEEE Transactions on Wireless Communications, pp. 660 - 670.

[19] Heinzelman W., (2000) "Application-Specific Protocol Architectures for Wireless Networks", $\mathrm{PhD}$ Thesis, Massachusetts Institute of Technology

[20] Cordina M, Debono C.J. "Increasing Wireless Sensor Network Lifetime through the Application of SOM neural networks.” ISCCSP, IEEE, Malta, 2008, pp. 467-471

[21] Mei, L., Haihao, Li., Shen, Y., Fan, J., Huang, SH., (2009) "Elastic neural network method for multi-target tracking task allocation in wireless sensor network", Computers and Mathematics with Applications, Vol. 57, Issue .11-12, pp. 1822--1828

[22] Yun, S.U., Youk, Y.S, Kim S.H. (2007) "Study on Applicability of Self-Organizing Maps to Sensor Network.” International Symposium on Advanced Intelligent Systems, Sokcho, Korea

[23] Mohamed, W.K., Mirza, O., Kawtharani, J. (2009) "BARC: A Battery Aware Reliable Clusteringalgorithm for sensor networks" Journal of Network and Compute Applications 32(6), pp.1183-1193

[24] Park, I., Takeshi, M., (2007) "Energy Reduction in Wireless Sensor Networks through Measurement Estimation with Second Order Recurrent Neural Networks", IEEE Third International Conference on Networking and Services (ICNS)

[25] Shen, Y., Guo, B., (2008) "Wavelet Neural Network Approach for Dynamic Power Management in Wireless Sensor Networks", International Conference on Embedded Software and Systems (ICESS2008), pp.376-381

[26] Smith, D., Singh, S. (2006) "Approaches to Multisensor Data Fusion in Target Tracking: A Survey”, IEEE TRANSACTIONS ON KNOWLEDGE AND DATA ENGINEERING, Vol. 18, No. 12, pp.

[27] H. Martı́nez Barbera’, A. Go'mez Skarmeta, M. Zamora Izquierdo, and J. Botia’ Blaya (2000), "Neural Networks for Sonar and Infrared Sensors Fusion", Proc. Third Int'1 Conf. Information Fusion, vol. 2, pp. WEB4/18-WEB4/25. 
International Journal of Computer Science \& Engineering Survey (IJCSES) Vol.1, No.1, August 2010

[28] M. Buchberger, K.W. Jorg, and E. von Puttkamer (1993) "Laser Radar and Sonar Based World Modelling and Motion Control for Fast Obstacle Avoidance of the Autonomous Mobile Robot MOBOTIV" Proc. IEEE Int'l Conf. Robotics and Automation, pp. 534-539.

[29] C.C. Chang and K.T. Song, (1996) "Ultrasonic Sensor Data Integration and Its Application to Environment Perception”, J. Robotic Systems, vol. 13, no. 10, pp. 663-677

[30] J.D. Courtney and A.K. Jain, (1994) "Neural Network Learning of Variable Grid-Based Maps for the Autonomous Navigation of Robots," Proc. IEEE Int'1 Conf. Robotics and Automation, pp. $40-45$

[31] V. Santos, J. Gonzalves, and F. Vaz, (1994) "Perception Maps for the Local Navigation of a Mobile Robot: A Neural Network Approach," IEEE Int'l Conf. Robotics and Automation, pp. 2193-2198,

[32] S. Thrun, (1998) "Learning Metric-Topological Maps for Indoor Mobile Robot Navigation", Artificial Intelligence, vol. 99, no. 1, pp. 21-71.

[33] M. Winter and G. Favier, (1999) “A Neural Network for Data Association”, Proc. 1999 IEEE Int'1 Conf. Acoustics, Speech, and Signal Processing, vol. 2, pp. 1041-1044

[34] S.P. Chaudhuri and S. Das, (1990) "Neural Networks for Data Fusion”, Proc. IEEE Int'l Conf. Systems Eng

[35] J. Moody and C. Darken, (1989) "Fast Learning in Networks of Locally Tuned Processing Units," Neural Computation, vol. 1, pp. 281-294

[36] T. Ash, (1989)“Dynamic Node Creation in Back propagation Networks”, technical report, Inst. for Cognitive Science, Univ. of California,San Diego

[37] Xue, W., Sheng, W., Ma, J.J., Bi, D.W., (2008) “Energy-efficient Organization of Wireless Sensor Networks with Adaptive Forecasting” Sensors 2008, vol. 8, pp. 2604-2616

[38] Mei, L., Haihao, L., Shen, Y., Fan, J., Huang, S.H., (2009) "Elastic neural network method for multi-target tracking task allocation in wireless sensor network", Computers and Mathematics with Applications 57(11-12), pp.1822-1828

[39] Feng, X., Xu, Z.H. (2009) “A Neural Data Fusion Algorithm for Wireless Sensor Networks”, Pacific-Asia Conference on Circuits, Communications and Systems, pp. 54-57

[40] Chung, Y.N., Chong, C.Y., Bar-Shalom, Y. (1986) "Joint Probabilistic data and association Distributed sensor Networks", IEEE Trans. Automa. Contr. AC-31, pp.889-897

[41] Sengupta, D., Iltis, R.A. (1989) "Neural solution to Multitarget Tracking Data Association Problem” IEEE Trans. Aerosp. Electron. Syst. 25, pp.86-108

[42] Salcedo-Sanz, S., Yao, X. (2004) "A hybrid Hopfield network-Genetic algorithm approach for the terminal assignment problem”, IEEE Transactions on Systems, Man, and Cybernetics PartB: Cybernetics 34(6), pp.2343-2353

[43] Ye M, Li C. F, Chen G. H, Wu J. (2005), "EECS: An Energy Efficient Clustering Scheme in Wireless Sensor Networks. " In: Proceedings of IEEE Int'l Performance Computing and Communications Conference (IPCCC), pp. 535-540.

[44] Enami, N, Askari Moghadam R. (2010) "Energy Based Clustering Self Organizing Map Protocol for Extending Wireless Sensor Networks Lifetime and Coverage" Canadian Journal on Multimedia and Wireless networks, AMPublishers, Vol.1, No.4, August 2010, pp.42-54.

[45] Intanagonwiwat C, Govindan R, Estrin D. (2000) “Directed Diffusion: a Scalable and Robust Communication Paradigm for Sensor Networks” Proc. ACM Mobi-Com, Boston, MA, pp. 5667.

[46] Enami,N and Dadashtabar, K.,(2009) "Query Processing Architecture in Wireless Sensor Network”, 3rd IC4I National Conference Proceedings, Tehran, Iran, pp. 315-326. 